\begin{tabular}{|c|c|}
\hline Title & Spectroscopic Photoresponse of the Passive Film Formed on Iron \\
\hline Author(s) & A zumi, Kazuhisa; Ohtsuka, Toshiaki; Sato, Norio \\
\hline Citation & $\begin{array}{l}\text { J. Electrochemical Society, 133(7), 1326-1328 } \\
\text { https://doi.org/10.1149/1.2108863 }\end{array}$ \\
\hline Issue Date & 1986-07 \\
\hline Doc URL & http:/hdl.handle.net/2115/62197 \\
\hline Rights & $\begin{array}{l}\text { (1) The Electrochemical Society, Inc. 1986. All rights reserved. Except as provided under U.S. copyright law, this work } \\
\text { may not be reproduced, resold, distributed, or modified without the express permission of The Electrochemical Society } \\
\text { (ECS). The archival version of this work was published in J. Electrochem. Soc. } 1986 \text { volume 133, issue 7, 1326-1328. }\end{array}$ \\
\hline Type & article \\
\hline File Information & J.Electrochem.Soc.133,1326-1329(1986), Spectroscopic photo respnse --iron.pdf \\
\hline
\end{tabular}

Instructions for use 


\title{
Spectroscopic Photoresponse of the Passive Film Formed on Iron
}

\author{
Kazuhisa Azumi, Toshiaki Ohtsuka, and Norio Sato* \\ Faculty of Engineering, Hokkaido University, Sapporo, Japan
}

\begin{abstract}
Spectroscopic photoresponse of the passive film formed on iron in neutral borate solution was studied. The spectroscopic three parameter refiectometry and the photoelectrochemical technique were employed to obtain the spectra of absorption coefficient and photocurrent. From these spectra, a conversion efficiency, $\eta$, from the flux of photons absorbed in the film to the photocurrent was calculated as a function of photon energy. The result shows that the photoexcitation process of electron-hole-pair formation includes two transition mechanisms; a direct allowed transition and an indirect allowed transition. The direct transition, which occurs in the photon energy higher than $2.6 \mathrm{eV}$, is attributed to the excitation between the valence (O-2p) and the conduction (Fe-3d) bands of iron oxide, whereas the indirect transition, which occurs in the photon energy higher than $0.8 \mathrm{eV}$, is associated with electronic levels of impurities or d-d transition in the passive film. The maximum value of $\eta$ observed at a photon energy of $3.1 \mathrm{eV}$ is about $20 \%$, and this high efficiency suggests that the high electric field exists in the passive film of iron.
\end{abstract}

Passivity of metals has been studied intensively for its industrial importance. Particularly, the electronic property of the passive film on metals has been investigated in recent years by means of optical spectroscopic techniques. Oshe et al. (1) measured the photovoltage and Whilhelm et al. (2) and Stimming (3) measured the photocurrent to examine the semiconductive property of the passive film on iron. These authors have found from their studies that the passive film on iron is a semiconductor of n-type. Recently, Cahan et al. (4) employed spectroscopic ellipsometry to measure the optical constant of the passive film formed on iron to reveal the electronic energy band structure of the film. There have also been a number of studies on the optical and electrochemical properties of iron oxides and oxide-covered iron electrodes in relation to the solar energy conversion cells, which require semiconductor electrodes possessing a suitable bandgap energy and corrosion resistance (5-7).

In this paper, we measure the spectroscopic photoinduced current of the passive film and discuss the photoinduced current conversion efficiency of the absorbed light. From the spectra of the conversion efficiency and of the optical constant, we suggest a band structure of the passive film.

\section{Experimental}

The iron electrode was prepared from a plate of Ferrvac-E iron of $99.99 \%$ purity. The exposed area of the electrode to the solution was ca. $0.8 \mathrm{~cm}^{2}$ for three parameter (3P) reflectometry and ca. $0.2 \mathrm{~cm}^{2}$ for photocurrent measurement. One side of the electrode was polished finally with $0.05 \mu \mathrm{m}$ alumina abrasive and washed in ethyl alcohol by using an ultrasonic cleaner.

The solution was a mixture of $0.30 \mathrm{~mol} \cdot \mathrm{dm}^{-3} \mathrm{H}_{3} \mathrm{BO}_{4}$ and $0.075 \mathrm{~mol} \cdot \mathrm{dm}^{-3} \mathrm{Na}_{2} \mathrm{~B}_{4} \mathrm{O}_{7}$ prepared from doubly distilled water and reagents of analytical grade, which were deaerated before use with purified nitrogen gas. All the experiments were performed at a constant temperature of $298 \pm 1 \mathrm{~K}$.

The counterelectrode was a platinum plate, and the reference electrode was a saturated calomel electrode (SCE). The electrode potential scale in this work is based on the reversible hydrogen electrode potential at the same solution (RHE).

The theoretical background and the equipment of the $3 P$-reflectometry have been described in previous papers (8-10). The equipment essentially consisted of a $\mathrm{P}$ (polarizer)-S(sample)-A(analyzer) configuration. In this technique, the relative reflectivity changes of the polarized light reflected at the specimen surface, $(\Delta R / R)$, were measured between the film-free and the film-covered surfaces at three polarization states of light to obtain the three measurable parameters.

$$
(\Delta R / R)=\left(I^{\mathrm{o}}-I\right) / I^{\mathrm{o}}
$$

*Electrochemical Society Active Member. where $I$ is the intensity of the reflection light at the filmcovered surface and $I^{\circ}$ at the film-free surface. The first two $(\Delta R R)$ values correspond to the reflectivity changes for $\mathrm{p}$ - and s-polarized lights. For the measurement of these $(\Delta R / R)$ values the azimuth of $A$ is fixed at $0^{\circ}$ and $90^{\circ}$, respectively, for $p$ - and s-components. For the measurement of an additional $(\Delta R / R)$ the azimuth of $P$ and $A$ are fixed at angles not equal to $0^{\circ}, 90^{\circ}, 180^{\circ}$, and $270^{\circ}$, and the resulting $(\Delta R / R)$ becomes a function of change in the phase difference between $\mathrm{p}$ - and s-polarized lights. These independent measurable three $(\Delta R / R)$ values can be used to evaluate three optical unknowns of the passive film, complex refractive index $N_{2}=n_{2}-i k_{2}$ and thickness $d$. Complex refractive index $N_{3}=n_{3}-i k_{3}$ of the substrate was estimated from the rotating analyzer ellipsometry technique for the film-free surface at each measurement wavelength (10). Measurements were conducted by using monochromatic light in the wavelength range between 340 and $660 \mathrm{~nm}$ at the incident angle of $55.07^{\circ}$.

The equipment of the photocurrent measurements is shown in Fig. 1. The light source of a $450 \mathrm{~W}$ xenon lamp and optical bandpass filters were used to obtain 25 sets of monochromatic light in the wavelength range from 276 to $823 \mathrm{~nm}$. The light beam was periodically chopped ( $f$ $=830 \mathrm{~Hz}$ ) and focused onto the electrode surface in the electrochemical cell through an optical glass window at normal incidence. A lock in amplifier (NF Circuit Corporation Model NI-574) was used to measure the photocurrent. The absolute photon flux at each wavelength was checked with a radiometer (EG \& G Corporation, Model 580 ).

\section{Experimental Result}

The complex refractive index $N_{2}=n_{2}-i k_{2}$ of the passive film at each wavelength was calculated from the three $(\Delta R R)$ values by assuming that the passive film is homogeneous and isotropic. The result is shown in Fig. 2, where the passive film was formed on iron by potentiostatic $1 \mathrm{~h}$ oxidation at $1.6 \mathrm{~V}$ in $p \mathrm{H} 8.4$ borate solution. The

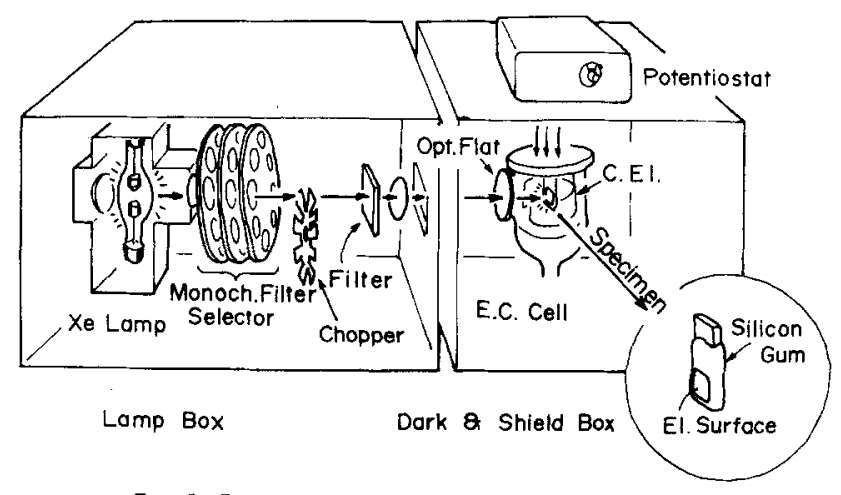

Fig. 1. Equipment for photocurrent measurements 


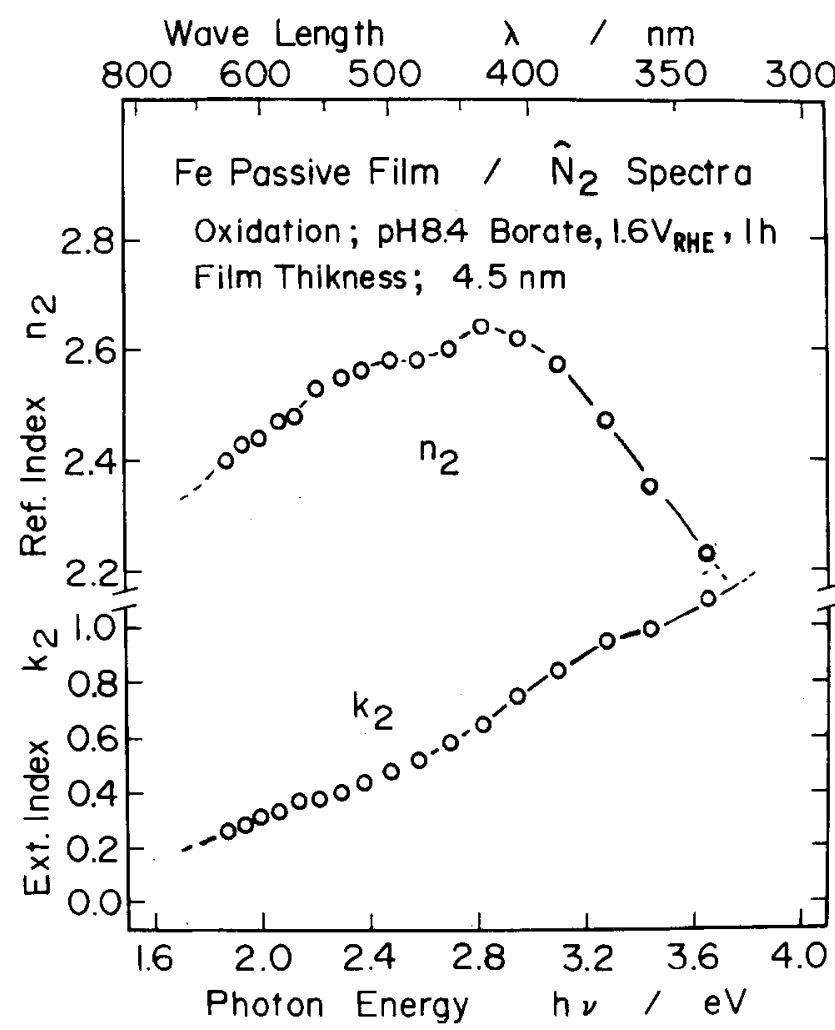

Fig. 2. Photon energy dependence of the refractive index, $n_{2}$, and the extinctive index, $k_{2}$, measured by three parameter reflectometry of the passive film formed on iron at $1.6 \mathrm{~V}_{\mathrm{RHE}}$ in $\mathrm{pH} 8.4$ borate solution. Film thickness was $4.5 \mathrm{~nm}$.

thickness of the film was $4.5 \mathrm{~nm}$, which was simultaneously calculated together with the complex refractive index. With increasing photon energy $(h \nu)$, the value of $n_{2}$ increases from 2.4 at $h \nu=1.9 \mathrm{eV}$ to 2.65 at $h \nu=2.8 \mathrm{eV}$ and then decreases from 2.65 at $h \nu=2.8 \mathrm{eV}$ to 2.2 at $h \nu=3.6$ $\mathrm{eV}$. The value of $k_{2}$ increases with photon energy from 0.2 at $h \nu=1.8 \mathrm{eV}$ to 1.1 at $h \nu=3.6 \mathrm{eV}$. The peak value of $n_{2}$ obtained at $2.8 \mathrm{eV}$ increases with the film formation potential in the passive region. The analogous result with Fig. 2 has previously been reported by Cahan et al. (4).

Photocurrent measurements were conducted for the iron passive film formed under the same condition as that shown in Fig. 2. The result of the photocurrent is shown in Fig. 3, where photocurrent $I_{\mathrm{p}}$ for unit watt is plotted as a function of photon energy. The magnitude of photocurrents measured of the specimen electrode was in the order of $\mu \mathrm{A}$. In Fig. 3, the inset indicates the photocurrent response measured by a monochromatic light beam at $\lambda=486 \mathrm{~nm}$ as a function of anodic potential. The photocurrent is found to have a maximum value near the potential of $1.6 \mathrm{~V}$. For the spectroscopic response the photocurrent $I_{\mathrm{p}}$ increases with increasing photon energy in the energy region from 1.8 to $3.0 \mathrm{eV}$ and decreases in the region higher than $3.5 \mathrm{eV}$, as shown in Fig. 3

\section{Discussion}

From the observed value of $k_{2}$, the light absorption coefficient $\alpha$ of the film can be calculated by using Eq. [2]

$$
2 \alpha=4 \pi k_{2} / \lambda
$$

where $\lambda$ is the wavelength. When the photon energy is close to the bandgap energy, $\alpha$ is known to have the following dependence on the photon energy (5-7)

$$
\alpha=(A / h \nu)\left(h \nu-E_{\mathrm{g}}\right)^{\mathrm{n}}
$$

where $A$ is a constant, $h \nu$ the photon energy, and $E_{\mathrm{g}}$ the bandgap energy. In Eq. [3], the photon absorption process corresponds to the direct allowed transition when $n=1 / 2$ and to the indirect allowed transition when $n=2$. If $h \nu$

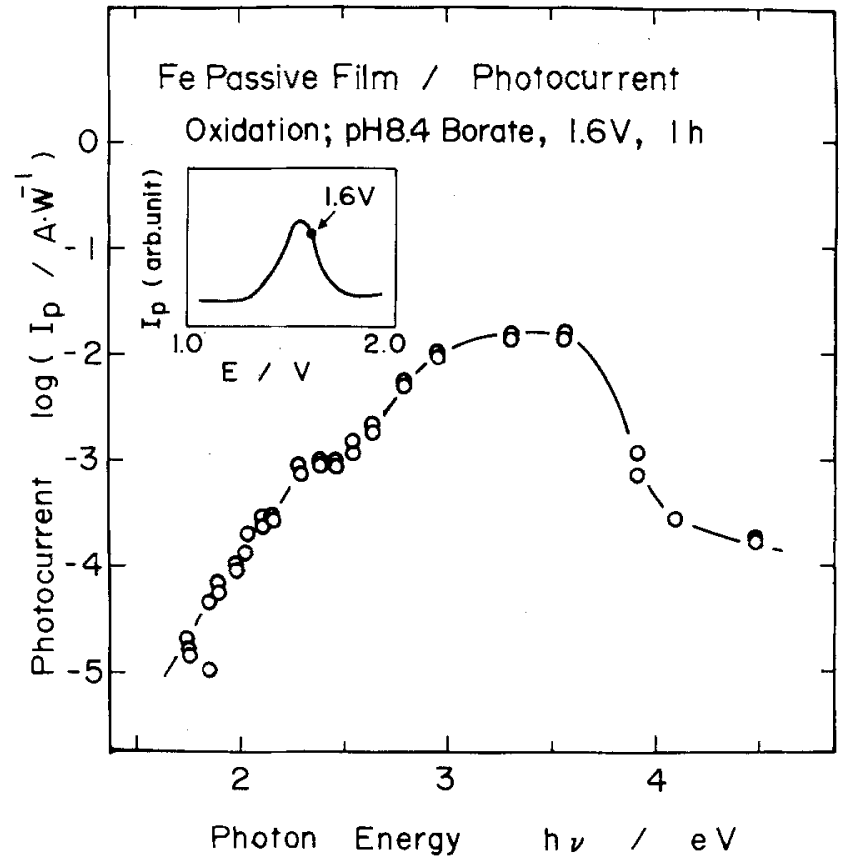

Fig. 3. Photon energy dependence of photocurrent $\left(\log I_{\mathrm{p}} / \mathrm{A} \cdot \mathrm{W}^{-1}\right)$ measured for the passive film formed on iron at $1.6 \mathrm{~V}_{\mathrm{RHE}}$ in $\mathrm{pH} 8.4$ borate solution. Photocurrent data wos corrected to the value per unit light power at each wavelength. An inset in the figure shows the dependence of photocurrent measured by monochromatic light of 485 $\mathrm{nm}$ on the film formotion potential.

$>>\left(h \nu-E_{\mathrm{g}}\right)$ is assumed, Eq. [3] can be approximated to Eq. [4]

$$
\alpha^{1 / \mathrm{n}}=A^{\prime}\left(h \nu-E_{\sharp}\right)
$$

Photon energy dependence of both $\alpha^{2}$ and $\alpha 1^{1 / 2}$ is shown in Fig. 4. For the direct allowed transition, a linear relationship between $\alpha^{2}(n=1 / 2)$ and $h \nu$ is seen in the region of photon energy higher than $2.6 \mathrm{eV}$. For the indirect allowed transition, a linear relationship between $\alpha^{1 / 2}(n=2)$ and $h \nu$ is seen in the region of photon energy lower than $2.6 \mathrm{eV}$.

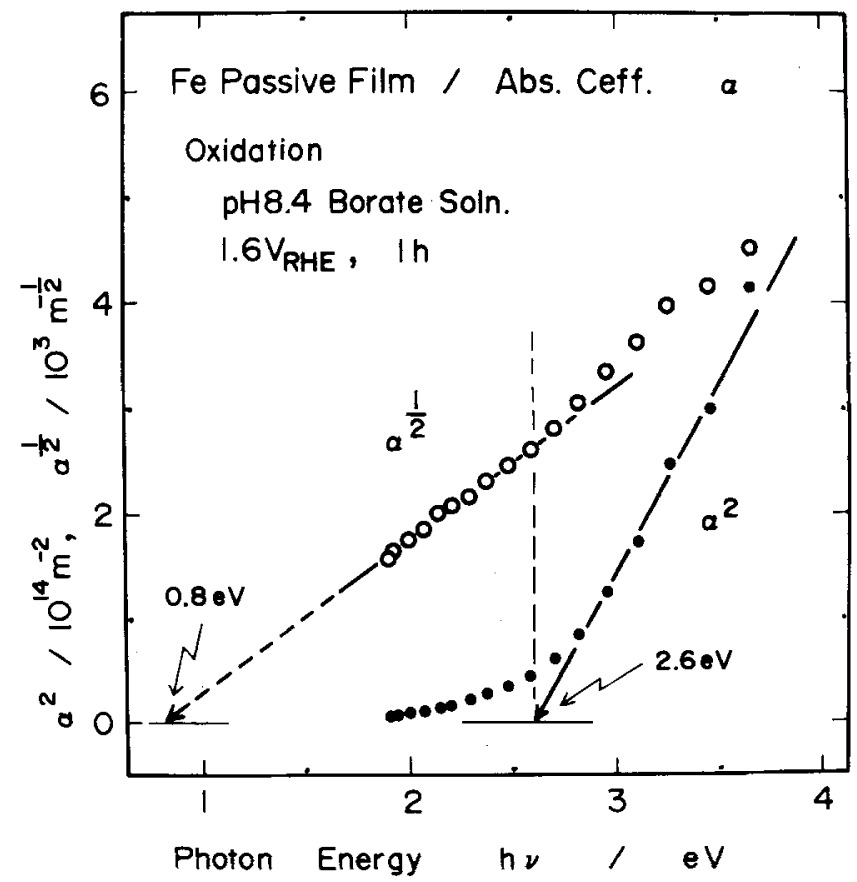

Fig. 4. Light absorption coefficient spectra for the passive film formed on iron at $1.6 \mathrm{~V}_{\mathrm{RHE}}$ in $\mathrm{pH} 8.4$ borate solution, calculated from extinctive index $k_{2}$ estimated by $3 P$-reflectometry. 
Extrapolation of the straight line of $\alpha^{2}$ to the zero point of $\alpha$ in Fig. 4 allows the bandgap energy to be estimated at $2.6 \mathrm{eV}$. This value of bandgap $2.6 \mathrm{eV}$ is fairly close to the values reported for ferric oxide and the passive film on iron $(2,6,7)$. This bandgap energy was found neither to depend on the film formation potential nor the solution $\mathrm{pH}$.

The straight line of $\alpha^{1 / 2}$ in the photon energy region lower than $2.6 \mathrm{eV}$ in Fig. 3 suggests the photoexcitation due to the indirect transition. From extrapolation of this straight line, the bandgap of this transition was found to be about $0.8 \mathrm{eV}$, which appeared to somewhat depend on the film formation condition.

Conversion efficiency of the incident photon to the photocurrent in the passive film formed on iron was reported by Whilhelm et al. to be on order of $1 \times 10^{-4}(2)$. This value of the conversion efficiency was obtained from the ratio of photogenerated carrier flow to the incident photon, which, however, does not take into account the effects of surface reflection of the incident light. From the complex refractive index and the thickness of the film, shown in Fig. 2, we can calculate using the Drude's and Fresnell's equations at normal incidence what amount of photon is absorbed by the film in the total incident photon flux. Further, if we assume the carrier generation efficiency to be $100 \%$, the theoretical photocurrent can be derived. From the theoretical photocurrent, $I_{\mathrm{p}}$ (theo), and measured photocurrent, $I_{\mathrm{p}}$ (meas), as shown in Fig. 3, the "real" conversion efficiency, $\eta$, can be calculated

$$
\eta=I_{\mathrm{p}} \text { (meas) } / I_{\mathrm{p}} \text { (theo) }
$$

Figure 5 shows the conversion efficiency $\eta$ against photon energy. The conversion efficiency $\eta$ is seen to be smaller than 0.05 in the region of photon energy lower than $2.6 \mathrm{eV}$ and increases steeply with photon energy reaching 0.2 at $3.0 \mathrm{eV}$. The result in Fig. 5 means that in the higher photon energy region, $80 \%$ of the carriers generated by photon excitation recombines with the majority carriers, while in the lower photon energy region, almost all the carriers generated by photon are annihilated by recombination.

From the results shown in Fig. 4 on the absorption processes and the results in Fig. 5 on the conversion effi-

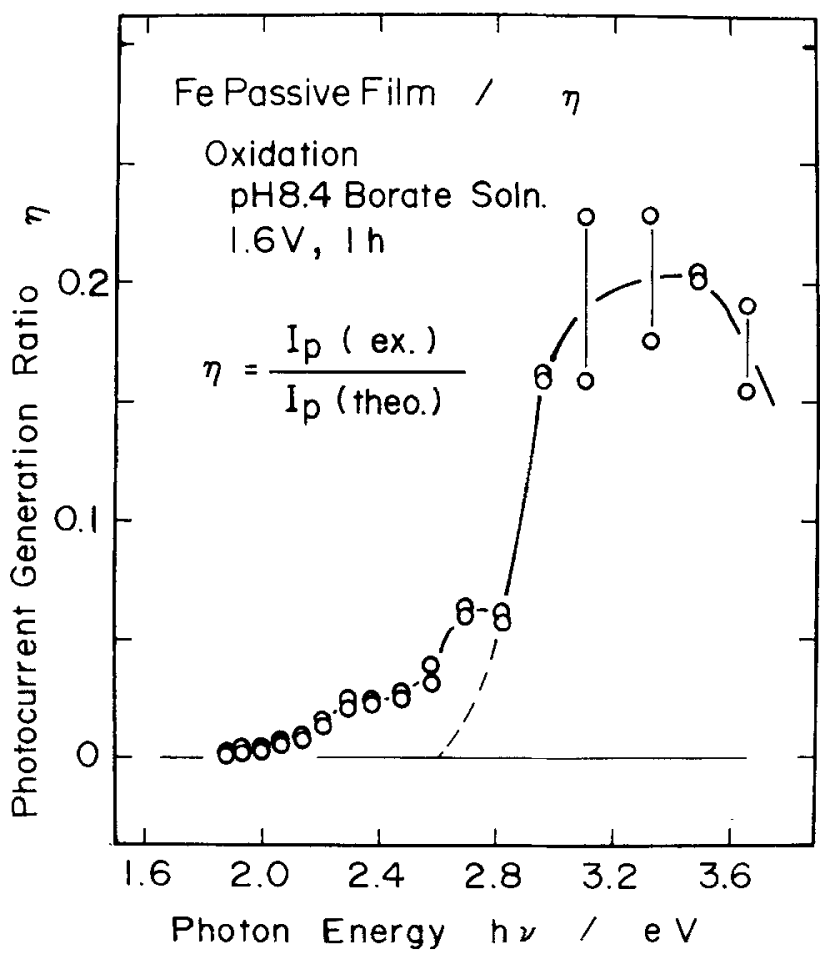

fig. 5. Photon energy dependence of effective photocarrier generation ratio $\eta$ to incident photon for the passive film formed on iron at $1.6 \mathrm{~V}_{\mathrm{BHE}}$ in $\mathrm{pH} 8.4$ borate solution, calculated from the data shown in Fig. 1 and 2. ciency, it follows that the low value of $\eta$ in the region of photon energy lower than $2.6 \mathrm{eV}$ corresponds to the indirect transition process. Thus, it is likely that the indirect transition scarcely generates the mobile carriers or that the recombination after excitation of electron-hole pairs generated by the indirect transition is very fast. This indirect transition process may be associated with the electron transition due to localized impurity levels in the passive film or with the $d$ - $d$ transition levels of iron oxide which has been found to have an absorption peak at 2.4 eV $(11,12)$

The higher value $\eta$ in the photon energy region exceeding $2.6 \mathrm{eV}$ can be attributed to the excitation between the valence $(\mathrm{O}-2 \mathrm{p})$ and the conduction (Fe-3d) bands of iron oxide which has been found to have an absorption peak at $3.2 \mathrm{eV}(11,12)$. Under the condition at $E=1.6 \mathrm{~V}$ and at $\lambda$ $=400 \mathrm{~nm}(h \nu=3.1 \mathrm{eV})$, for example, $11 \%$ of the photon incident to the electrode surface is absorbed, and $20 \%$ of the absorbed photon contributes to the photocurrent. This high value of $\eta$ under the anodic bias condition means that $1 / 5$ of the total holes excited in the space charge region of the n-type semiconductive film flows into the oxide-aqueous solution surface and another $4 / 5$ recombines with the electron of the conduction band. The high efficiency is probably due to the large electric field of about $3 \times 10^{8} \mathrm{~V} \cdot \mathrm{m}^{-1}$ in the passive film and the very small thickness of about $4.5 \mathrm{~nm}$ of the film. After excitation due to the incident photon, the carrier separation occurs rapidly, and the recombination will not be marked because of small distance for the moving carriers to the iron substrate or to the oxide/solution interface.

In summary, it has been found in this work on the spectroscopic photoresponse of the iron passive film that there are two electron transition processes for excitation of electron due to photon illumination in the visual light region. The process that occurs in the lower energy region corresponds to the indirect allowed process and its conversion efficiency for photocurrent is low. The other process that occurs in the higher energy region corresponds to the direct allowed process and its conversion efficiency for photocurrent is relatively high.

Manuscript submitted April 29, 1984; revised manuscript received Jan: 22, 1986 .

Hokkaido University assisted in meeting the publication costs of this article.

\section{REFERENCES}

1. K. E. Oshe and I. L. Rosenfeld, Electrokhimiya, 4, 1200 (1968).

2. S. M. Whilhelm, K. S. Yun, L. W. Ballenger and N. Hackerman, This Journal, 126, 419 (1979); S. M. Whilhelm and N. Hackerman, ibid., 128, 1668 (1981).

3. U. Stimming in "Passivity of Metals and Semiconductors," M. Froment, Editor, p. 477, Elsevier, Amsterdam (1983).

4. C. T. Chen and B. D. Cahan, This Journal, 129, 17 (1982); D. J. Wheeler, B. D. Cahan, C. T. Chen, and E. Yeager, in "Passivity of Metals" R. P. Frankenthal and J. Kruger, Editors, p. 546, The Electrochemical Society, Inc., Princeton, NJ' (1978).

5. M. P. Dare-Edwards, J. B. Goodenough, A. Hamnett, and P. R. Trevellick, J. Chem. Soc., Faraday Trans. 1, 79, 2027 (1983).

6. J. E. Turner, M. Hendewerk, J. Permerter, D. Neiman, and G. A. Somorjai, This Journal, 131, 1777 (1984).

7. M. A. Butler, D. S. Ginley, and E. Eibshutz, J. Appl. Phys., 48, 3070 (1977).

8. T. Ohtsuka and K. E. Heusler, J. Electroanal. Chem., 100, 319 (1979); K. E. Heusler and T. Ohtsuka, Surf. Sci., 101, 194 (1980).

9. T. Ohtsuka, K. Azumi, and N. Sato, in "Passivity of Metals and Semiconductors," M. Froment Editor, p. 199, Elsevier, Amsterdam (1983); T. Ohtsuka, K. Azumi, and N. Sato, J. de Phys., C10, 191 (1983).

10. T. Ohtsuka, K. Azumi, and N. Sato, Reports of the Asahi Glass Foundation For Industrial Technology, 41, 197 (1982).

11. R. F. G. Gardner, F. Sweett, and D. W. Tanner, J. Phys. Chem. Solids, 24, 1183 (1963).

12. N. C. Debnath and A. B. Andersen, This Journal, 129, 2169 (1982). 